\title{
Quantum Mechanics of Damped Systems
}

\author{
Dariusz Chruściński* \\ Department of Mathematics and Statistics \\ University of Calgary \\ Calgary, Alberta, Canada
}

\begin{abstract}
We show that the quantization of a simple damped system leads to a self-adjoint Hamiltonian with a family of complex generalized eigenvalues. It turns out that they correspond to the poles of energy eigenvectors when continued to the complex energy plane. Therefore, the corresponding generalized eigenvectors may be interpreted as resonant states. We show that resonant states are responsible for the irreversible quantum dynamics of our simple model.
\end{abstract}

Mathematical Subject Classifications (2000): 46E10, 46F05, 46N50, 47A10.

Key words: quantum mechanics, distributions, spectral theorem, Gelfand triplets.

* On the leave from Institute of Physics, Nicolaus Copernicus University, ul. Grudziądzka 5/7, 87-100 Toruń, Poland 


\section{Introduction}

Standard textbooks on quantum mechanics investigate mainly the Hamiltonian system, i.e. by a quantum system one usually means a Hilbert space $\mathcal{H}$ which describes physical quantum states and a self-adjoint operator (Hamiltonian) in $\mathcal{H}$ which governs dynamics of the system. However, most of the classical systems are not Hamiltonian and the quantum mechanics of such systems is poorly understood. In the present paper we are going to investigate one of the simplest non-Hamiltonian system corresponding to a damped motion in one dimension:

$$
\dot{x}=-\gamma x
$$

where $x \in \mathbb{R}$, and $\gamma>0$ stands for the damping constant. Classically, the damping behavior is described by the exponential law

$$
x(t)=e^{-\gamma t} x .
$$

As is well known [1] (see also [2]), within the standard Hilbert space formulation of quantum mechanics there is no room for such a behaviour on a quantum level. Therefore, in order to deal with this problem, we shall use the rigged Hilbert space approach to quantum mechanics which generalizes the standard Hilbert space version $[3,4,5]$. A rigged Hilbert space (or a Gelfand triplet) is a collection of spaces $[6,7]$ :

$$
\Phi \subset \mathcal{H} \subset \Phi^{\prime},
$$

where $\mathcal{H}$ is a Hilbert space, $\Phi$ is a dense nuclear subspace of $\mathcal{H}$, and $\Phi^{\prime}$ denotes its dual, i.e. the space of continuous functionals on $\Phi$ (see section 2 for a brief review).

The quantization of our simple model (1.1) leads to a self-adjoint Hamiltonian $\widehat{H}$ in $\mathcal{H}=L^{2}(\mathbb{R})$. Interestingly, $\widehat{H}$ being self-adjoint, gives rise to the family of generalized complex eigenvalues. Clearly, these eigenvalues are not elements of the spectrum $\sigma(\widehat{H})=(-\infty, \infty)$. The corresponding eigenvectors do not belong to $L^{2}(\mathbb{R})$ but to $\Phi^{\prime}$ for an appropriately chosen $\Phi$. We show that these complex eigenvalues have many remarkable properties analogous to the point spectrum of a self-adjoint operator. In particular, they give rise to the spectral decomposition of $\widehat{H}$. Moreover, they are closely related to the continuous spectrum of $\widehat{H}$. It turns out that they correspond to the poles of the energy eigenvectors $\psi^{E}$ when continued to the complex energy plane [8]. Physicists usually called the corresponding eigenvectors resonant states $[3,9,10]$ (see also [11]). It is widely believed that resonant states are responsible for the irreversible dynamics of physical systems (see e.g. recent collection of papers [3]). Indeed, it is true in our simple model. To see this we construct two Gelfand triples:

$$
\Phi_{ \pm} \subset L^{2}(\mathbb{R}) \subset \Phi_{ \pm}^{\prime}
$$

such that $\Phi_{+} \cap \Phi_{-}=\{\emptyset\}$. Obviously, the time evolution is perfectly reversible when considered

on $L^{2}(\mathbb{R})$. It is given by the 1 -parameter group of unitary transformations $U(t)=e^{-i \hat{H} t}$. However, when restricted to $\Phi_{ \pm}$, it defines only two semigroups: $U(t \geq 0)$ on $\Phi_{-}$, and $U(t \leq 0)$ on $\Phi_{+}$. Therefore, the evolution on $\Phi_{ \pm}$is irreversible. This irreversibility is caused by quantum damping, or, equivalently, by the presence of resonances. 


\section{Rigged Hilbert space}

Consider a rigged Hilbert space, i.e. the following collection (Gelfand triplet):

$$
\Phi \subset \mathcal{H} \subset \Phi^{\prime}
$$

where $\mathcal{H}$ is a Hilbert space with the standard norm topology $\tau_{\mathcal{H}}, \Phi$ is a topological vector space with a topology, $\tau_{\Phi}$, stronger than $\tau_{\mathcal{H}}$, and $\Phi^{\prime}$ is the dual space of continuous linear functionals on $\Phi[6,7]$. We denote the action of $\Phi^{\prime}$ on $\Phi$ using Dirac notation, i.e. for any $\phi \in \Phi$ and $F \in \Phi^{\prime}$

$$
\langle\phi \mid F\rangle:=F(\phi) .
$$

Any self-adjoint operator $\widehat{A}$ in $\mathcal{H}$ may be extended to an operator on $\Phi^{\prime}$ :

$$
\widehat{A}: \Phi^{\prime} \rightarrow \Phi^{\prime},
$$

by

$$
\langle\phi \mid \widehat{A} F\rangle:=\langle\widehat{A} \phi \mid F\rangle
$$

Now, if for any $\phi \in \Phi$

$$
\left\langle\phi \mid \widehat{A} F_{\lambda}\right\rangle=\lambda\left\langle\phi \mid F_{\lambda}\right\rangle
$$

then $F_{\lambda} \in \Phi^{\prime}$ is called a generalized eigenvector corresponding to a generalized eigenvalue $\lambda$. Omitting $\phi$ one simply writes:

$$
\widehat{A}\left|F_{\lambda}\right\rangle=\lambda\left|F_{\lambda}\right\rangle
$$

Note, that a generalized eigenvalue $\lambda$ may be complex. Now, if the spectrum of $\widehat{A}$

$$
\sigma(\widehat{A})=\sigma_{p}(\widehat{A}) \cup \sigma_{c}(\widehat{A}) \subset \mathbb{R},
$$

with $\sigma_{p}(\widehat{A})=\left\{\lambda_{1}, \lambda_{2}, \ldots\right\}$, then the Gelfand-Maurin theorem [6, 7] implies the following spectral decompositions:

$$
\mathbb{1}_{\Phi}=\sum_{n}\left|F_{n}\right\rangle\left\langle F_{n}\left|+\int_{\sigma_{c}(\widehat{A})} d \lambda\right| F_{\lambda}\right\rangle\left\langle F_{\lambda}\right|,
$$

and of $\widehat{A}$ itself:

$$
\widehat{A}=\sum_{n} \lambda_{n}\left|F_{n}\right\rangle\left\langle F_{n}\left|+\int_{\sigma_{c}(\widehat{A})} d \lambda \lambda\right| F_{\lambda}\right\rangle\left\langle F_{\lambda}\right| .
$$

This way the rigged Hilbert space approach fully justifies the standard Dirac notation.

The choice of $\Phi$ depends on the particular problem one deals with. In the present paper we shall consider the following functional spaces: $\mathcal{D}$ - the space of $C^{\infty}(\mathbb{R})$ functions with 
compact supports equipped with the convex Schwartz topology [12], $\mathcal{S}$ - the space of $C^{\infty}(\mathbb{R})$ functions vanishing at infinity faster than any polynomial [12]. Moreover, let us define

$$
\mathcal{Z}:=\{F[\phi] \mid \phi \in \mathcal{D}\}
$$

where $F[\phi]$ denotes the Fourier transform of $\phi$. It turns out [13] that $\mathcal{Z}$ is isomorphic to the space of entire functions of fast decrease along $\mathbb{R}$. More precisely, let

$$
F_{L}[\phi](z):=\frac{1}{\sqrt{2 \pi}} \int_{-\infty}^{\infty} e^{i z x} \phi(x) d x
$$

be the Fourier-Laplace transform of $\phi \in \mathcal{D}$. One proves $[12,13]$ the following

Theorem 1 (Paley-Wiener-Schwartz) Let $a>0$. An entire function $U(z)$ is a FourierLaplace transform of a function $u \in \mathcal{D}$ with support

$$
\operatorname{supp}(u)=\{x \in \mathbb{R}|| x \mid \leq a\},
$$

if and only if

$$
|z|^{n}|U(z)| \leq C_{n} e^{a|\operatorname{Im} z|}, \quad n=1,2, \ldots .
$$

Now, for $z=x \in \mathbb{R}$, i.e. $\operatorname{Im} z=0, F_{L}[\phi]=F[\phi]$, and the above theorem implies

$$
|x|^{n}|F[\phi](x)| \leq C_{n}, \quad n=1,2, \ldots .
$$

Clearly, $\mathcal{Z} \cap \mathcal{D}=\{\emptyset\}$. Moreover, one has

$$
\mathcal{D} \subset \mathcal{S} \subset L^{2}(\mathbb{R})
$$

and

$$
\mathcal{Z} \subset \mathcal{S} \subset L^{2}(\mathbb{R})
$$

and both $\mathcal{D}$ and $\mathcal{Z}$ are dense in $\mathcal{S}$. One proves [13] that the Fourier transformation which defines the unitary operator

$$
F: L^{2}(\mathbb{R}) \longrightarrow L^{2}(\mathbb{R})
$$

establishes an isomorphism between $\mathcal{D}$ and $\mathcal{Z}$.

\section{Quantization of damped systems}

Let us quantize a classical damped system described by (1.1). Clearly this system is not Hamiltonian. However, it is well known (cf. [14]) that any dynamical system may be rewritten in a Hamiltonian form. Consider a dynamical system on $n$-dimensional configuration space $Q$ :

$$
\dot{x}=X(x)
$$


where $X$ is a vector field on $Q$. Now, define the following Hamiltonian on the cotangent bundle $\mathcal{P}=T^{*} Q$ :

$$
H\left(\alpha_{x}\right):=\alpha_{x}(X(x)),
$$

where $\alpha_{x} \in T_{x}^{*} Q$. Using canonical coordinates $\left(x^{1}, \ldots, x^{n}, p_{1}, \ldots, p_{n}\right)$ one obtains:

$$
H(x, p)=\sum_{k=1}^{n} p_{k} X^{k}(x),
$$

where $X^{k}$ are components of $X$ in the coordinate basis $\partial / \partial x^{k}$. The corresponding Hamilton equations take the following form:

$$
\begin{aligned}
& \dot{x}^{k}=\left\{x^{k}, H\right\}=X^{k}(x), \\
& \dot{p}_{k}=\left\{p_{k}, H\right\}=-\sum_{l=1}^{n} p_{l} \frac{\partial X^{l}(x)}{\partial x^{k}},
\end{aligned}
$$

for $k=1, \ldots, n$. In the above formulae $\{$,$\} denotes the canonical Poisson bracket on T^{*} Q$ :

$$
\{F, G\}=\sum_{k=1}^{n}\left(\frac{\partial F}{\partial x^{k}} \frac{\partial G}{\partial p_{k}}-\frac{\partial G}{\partial x^{k}} \frac{\partial F}{\partial p_{k}}\right) .
$$

Clearly, the formulae (3.4) reproduce our initial dynamical system (3.1) on $Q$.

Let us apply the above procedure to the damped system (1.1). One obtains for the Hamiltonian

$$
H(x, p)=-\gamma x p
$$

and hence the corresponding Hamilton equations

$$
\dot{x}=-\gamma x, \quad \dot{p}=\gamma p,
$$

give rise to the following Hamiltonian flow on $\mathbb{R}^{2}$ :

$$
(x, p) \longrightarrow\left(e^{-\gamma t} x, e^{t \gamma} p\right) .
$$

Now, the quantization is straightforward: one has for the Hilbert space $\mathcal{H}=L^{2}(\mathbb{R})$, and for the Hamiltonian

$$
\widehat{H}=-\frac{\gamma}{2}(\widehat{x} \widehat{p}+\widehat{p} \widehat{x})
$$

It is evident that (3.10) defines a symmetric operator on $L^{2}(\mathbb{R})$. In section 4 we show that $\widehat{H}$ is self-adjoint and hence it gives rise to a well defined quantum mechanical problem. ${ }^{1}$

\footnotetext{
${ }^{1}$ Actually, this Hamiltonian is well known in quantum optics in connection with the squeezed states of light [15]. Introducing $\widehat{a}$ and $\widehat{a}^{*}$ :$$
\widehat{x}=\frac{\widehat{a}+\widehat{a}^{*}}{\sqrt{2}}, \quad \widehat{p}=\frac{\widehat{a}-\widehat{a}^{*}}{\sqrt{2} i},
$$

the Hamiltonian (3.10) may be rewritten as follows:

$$
\widehat{H}=\frac{\gamma}{2 i}\left(\widehat{a}^{* 2}-\widehat{a}^{2}\right)
$$

which is exactly a generator of squeezing. 
Let us observe that performing the canonical transformation

$$
x=\frac{1}{\sqrt{2 \gamma}}(\gamma X-P), \quad p=\frac{1}{\sqrt{2 \gamma}}(\gamma X+P),
$$

the classical Hamiltonian (3.10) takes the following form:

$$
\widehat{H}=\frac{1}{2}\left(\widehat{P}^{2}-\gamma^{2} \widehat{X}^{2}\right)
$$

that is, it corresponds to the so called reversed harmonic oscillator. This system was analyzed in $[16]$ and recently in $[17,18,19]$ (see also $[20,21]$ ).

\section{Properties of the Hamiltonian}

Let us investigate the basic properties of the Hamiltonian defined in (3.10).

Proposition 1 The operator $\widehat{H}=-\frac{\gamma}{2}(\widehat{x} \widehat{p}+\widehat{p} \widehat{x})$ is self-adjoint in $L^{2}(\mathbb{R})$.

Proof. To prove that $\widehat{H}$ is self-adjoint we show that $e^{-i \widehat{H}}$ is unitary in $L^{2}(\mathbb{R})$. One has

$$
\widehat{H}=-\frac{\gamma}{2}(\widehat{x} \widehat{p}+\widehat{p} \widehat{x})=i \gamma\left(x \frac{d}{d x}+\frac{1}{2}\right) .
$$

Let us define

$$
U=e^{-i \widehat{H}}=e^{\gamma / 2} e^{\gamma x \partial_{x}}
$$

Clearly,

$$
U \psi(x)=e^{\gamma / 2} \psi\left(e^{\gamma} x\right)
$$

for any $\psi \in L^{2}(\mathbb{R})$. The operator $U$ defines an isometry:

$$
\begin{aligned}
\langle U \psi \mid U \phi\rangle & =\int_{-\infty}^{\infty} \overline{U \psi(x)} U \phi(x) d x=\int_{-\infty}^{\infty} e^{\gamma} \overline{\psi\left(e^{\gamma} x\right)} \phi\left(e^{\gamma} x\right) d x=\int_{-\infty}^{\infty} \overline{\psi(y)} \phi(y) d y \\
& =\langle\psi \mid \phi\rangle .
\end{aligned}
$$

Moreover, due to (4.3), $U$ is onto, and hence it is unitary in $L^{2}(\mathbb{R})$. Therefore, Stone's theorem implies that $\widehat{H}$ is self-adjoint (see e.g. [12]).

Obviously, $\widehat{H}$ is parity invariant:

$$
\mathbf{P} \widehat{H} \mathbf{P}^{-1}=\widehat{H},
$$

where the parity operator $P$ is defined by:

$$
\mathbf{P} \widehat{x} \mathbf{P}^{-1}=-\widehat{x}, \quad \mathbf{P} \widehat{p} \mathbf{P}^{-1}=-\widehat{p} .
$$


Now, let us turn to the time reversal operator $\mathbf{T}$. The theory invariant under the time reversal has the following property: if $\psi(t)$ is a solution of the Schrödinger equation given by

$$
\psi(t)=U(t) \psi
$$

with $U(t)=e^{-i \widehat{H} t}$, then $\mathbf{T} \psi$ evolves into

$$
(\mathbf{T} \psi)(-t)=U(t)(\mathbf{T} \psi)
$$

or, equivalently

$$
\mathbf{T}(U(t) \psi)=U(-t)(\mathbf{T} \psi),
$$

for any $\psi \in \mathcal{H}$. Now, following Wigner [22], $\mathbf{T}$ is either unitary or anti-unitary. If $\mathbf{T}$ is unitary, then (4.9) implies

$$
\mathbf{T} \widehat{H}+\widehat{H} \mathbf{T}=0
$$

It means that if

$$
\widehat{H} \psi^{E}=E \psi^{E},
$$

then

$$
\widehat{H} \mathbf{T} \psi^{E}=-E \mathbf{T} \psi^{E},
$$

that is, any eigenvector $\psi^{E}$ with the energy $E$ is accompanied by $\mathbf{T} \psi^{E}$ with energy $-E$. Usually, this case is excluded since one expects that the Hamiltonian is bounded from below. If this is the case, then $\mathbf{T}$ is anti-unitary and (4.9) implies:

$$
\mathbf{T} \widehat{H}-\widehat{H} \mathbf{T}=0 .
$$

However, the Hamiltonian defined in (3.10) is not bounded from below, and, as we show in section 6 its spectrum $\sigma(\widehat{H})=(-\infty, \infty)$. Therefore, we take $\mathbf{T}$ to be unitary in $L^{2}(\mathbb{R})$.

Proposition $\mathbf{2}$ The time reversal operator $\mathbf{T}$ is realized by the Fourier transformation:

$$
\mathbf{T} \psi:=F[\psi]
$$

i.e.

$$
F^{-1} \widehat{H} F \psi=-\widehat{H} \psi
$$

for all $\psi \in L^{2}(\mathbb{R})$. Moreover,

$$
\mathbf{T}^{2} \psi(x)=\mathbf{P} \phi(x)=\psi(-x) .
$$

Denoting by $\mathbf{C}$ the complex conjugation $\mathbf{C} \psi=\bar{\psi}$, one immediately finds

Proposition 3 The Hamiltonian (3.10) is CT and PCT invariant, i.e.

$$
[\widehat{H}, \mathbf{C T}]=[\widehat{H}, \mathbf{P C T}]=0 \text {. }
$$

Therefore, if

$$
\widehat{H} \psi^{E}=E \psi^{E},
$$

then

$$
\widehat{H} F\left[\overline{\psi^{E}}\right]=E F\left[\overline{\psi^{E}}\right] .
$$

Clearly, CT invariance does not produce any conserved quantity since $\mathbf{C T}$ is anti-unitary. 


\section{Complex eigenvalues}

Interestingly, $\widehat{H}$ being self-adjoint admits generalized eigenvectors with complex eigenvalues $[19,23,20,21]$. Let $f_{0}^{ \pm}$be distributions satisfying

$$
\widehat{x} f_{0}^{-}=0, \quad \widehat{p} f_{0}^{+}=0 .
$$

Clearly,

$$
f_{0}^{-}(x)=\delta(x), \quad f_{0}^{+}(x)=1
$$

Its easy to see that

$$
\widehat{H} f_{0}^{ \pm}= \pm i \frac{\gamma}{2} f_{0}^{ \pm}
$$

Let us define two families:

$$
f_{n}^{-}:=\frac{(-i)^{n}}{\sqrt{n !}} \widehat{p}^{n} f_{0}^{-}, \quad f_{n}^{+}:=\frac{1}{\sqrt{n !}} \widehat{x}^{n} f_{0}^{+} .
$$

One finds

$$
f_{n}^{-}(x)=\frac{(-1)^{n}}{\sqrt{n !}} \delta^{(n)}(x), \quad f_{n}^{+}(x)=\frac{x^{n}}{\sqrt{n !}}
$$

Moreover,

$$
\widehat{H} f_{n}^{ \pm}= \pm E_{n} f_{n}^{ \pm}
$$

where

$$
E_{n}:=i \gamma\left(n+\frac{1}{2}\right)
$$

Clearly, both $f_{n}^{-}$and $f_{n}^{-}$are tempered distributions, i.e. $f_{n}^{ \pm} \in \mathcal{S}^{\prime}$. Evidently, they are related by the Fourier transformation:

$$
F\left[f_{n}^{+}\right]=\sqrt{2 \pi} i^{n} f_{n}^{-}, \quad F\left[f_{n}^{-}\right]=\frac{i^{n}}{\sqrt{2 \pi}} f_{n}^{+} .
$$

Let us observe, that these two families of generalized eigenvectors have two remarkable properties:

$$
\int_{-\infty}^{\infty} f_{n}^{+}(x) f_{m}^{-}(x) d x=\delta_{n m}
$$

and

$$
\sum_{n=0}^{\infty} f_{n}^{+}(x) f_{n}^{-}\left(x^{\prime}\right)=\delta\left(x-x^{\prime}\right)
$$


These formulae remind one of the basic basic properties of proper (Hilbert space) eigenvectors: if $\widehat{A}$ is a self-adjoint operator in $\mathcal{H}$ and

$$
\widehat{A} \psi_{k}=\lambda_{k} \psi_{k}
$$

where $\psi_{k}$ are normalized vectors in $\mathcal{H}$, then

$$
\int \overline{\psi_{n}}(x) \psi_{m}(x) d x=\delta_{n m}
$$

and

$$
\sum_{n} \overline{\psi_{n}}(x) \psi_{n}\left(x^{\prime}\right) d x=\delta\left(x-x^{\prime}\right)
$$

Obviously, there is no complex conjugation in (5.9) and (5.10) since $f_{n}^{ \pm}$are real functions.

Now, for any $\phi \in \mathcal{Z}$ one has

$$
\phi(x)=\sum_{n} \frac{\phi^{(n)}(0)}{n !}(-1)^{n} x^{n}=\sum_{n} f_{n}^{+}(x)\left\langle f_{n}^{-} \mid \phi\right\rangle .
$$

On the other hand, for any $\phi \in \mathcal{D}$, its Fourier transform $F[\phi] \in \mathcal{Z}$, and

$$
\begin{aligned}
\phi(x) & =\frac{1}{\sqrt{2 \pi}} \int e^{i k x} F[\phi](k) d k=\frac{1}{\sqrt{2 \pi}} \int e^{i k x} \sum_{n} \frac{F[\phi]^{(n)}(0)}{n !}(-1)^{n} k^{n} d k \\
& =\sum_{n} F\left[f_{n}^{+}\right](x)\left\langle f_{n}^{-} \mid F[\phi]\right\rangle=\sum_{n} F\left[f_{n}^{+}\right](x)\left\langle F\left[f_{n}^{-}\right] \mid \phi\right\rangle \\
& =\sum_{n} f_{n}^{-}(x)\left\langle f_{n}^{+} \mid \phi\right\rangle .
\end{aligned}
$$

Hence, we have two spectral decompositions:

$$
|\phi\rangle=\sum_{n}\left|f_{n}^{+}\right\rangle\left\langle f_{n}^{-} \mid \phi\right\rangle \quad \text { in } \quad \mathcal{Z}
$$

and

$$
|\psi\rangle=\sum_{n}\left|f_{n}^{-}\right\rangle\left\langle f_{n}^{+} \mid \psi\right\rangle \quad \text { in } \quad \mathcal{D}
$$

In section 7 we derive (5.16) and (5.17) from the spectrum of $\widehat{H}$. So let us look for $\sigma(\widehat{H})$.

\section{Spectrum}

The Hamiltonian (3.10) has a continuous spectrum $\sigma(\widehat{H})=(-\infty, \infty)$. Since, the Hamiltonian (3.10) is parity invariant each generalized eigenvalue $E \in \mathbb{R}$ is doubly degenerated:

$$
\widehat{H} \psi_{ \pm}^{E}=E \psi_{ \pm}^{E}
$$


The above equation may be rewritten as the following differential equation for $\psi_{ \pm}^{E}$ :

$$
x \frac{d}{d x} \psi_{ \pm}^{E}(x)=-\left(i \frac{E}{\gamma}+\frac{1}{2}\right) \psi_{ \pm}^{E} .
$$

To solve (6.2) let us introduce the following distributions [13] (see also [24]):

$$
x_{+}^{\lambda}:=\left\{\begin{array}{ll}
x^{\lambda} & x \geq 0 \\
0 & x<0
\end{array}, \quad x_{-}^{\lambda}:=\left\{\begin{array}{cc}
0 & x \geq 0 \\
|x|^{\lambda} & x<0
\end{array},\right.\right.
$$

with $\lambda \in \mathbb{C}$ (basic properties of $x_{ \pm}^{\lambda}$ are collected in the Appendix). It is, therefore, clear that the generalized eigenvectors $\psi_{ \pm}^{E}$ may be written as follows:

$$
\psi_{ \pm}^{E}(x):=\frac{1}{\sqrt{2 \pi \gamma}} x_{ \pm}^{-(i E / \gamma+1 / 2)} .
$$

It turns out that $\psi_{ \pm}^{E}$ are well defined tempered distributions for all $E \in \mathbb{R}$. Actually, instead of $\psi_{ \pm}^{E}$ one may work with eigenvectors of the parity operator $\mathbf{P}$ :

$$
\begin{aligned}
\psi_{\text {even }}^{E} & =\frac{1}{\sqrt{2}}\left(\psi_{+}^{E}+\psi_{-}^{E}\right), \\
\psi_{\text {odd }}^{E} & =\frac{1}{\sqrt{2}}\left(\psi_{+}^{E}-\psi_{-}^{E}\right) .
\end{aligned}
$$

Obviously

$$
\mathbf{P} \psi_{\text {even }}^{E}=\psi_{\text {even }}^{E}, \quad \mathbf{P} \psi_{\text {odd }}^{E}=-\psi_{\text {odd }}^{E} .
$$

These distributions of definite parity are given by:

$$
\psi_{\text {even }}^{E}=\frac{1}{2 \sqrt{\pi \gamma}}|x|^{-(i E / \gamma+1 / 2)}, \quad \psi_{\text {odd }}^{E}=\frac{1}{2 \sqrt{\pi \gamma}} \operatorname{sign}(x)|x|^{-(i E / \gamma+1 / 2)},
$$

(see [13] and [24] for the properties of $|x|^{\lambda}$ and $\operatorname{sign}(x)|x|^{\lambda}$ ).

With the normalization used in (6.4) one proves [25] orthonormality:

$$
\int \overline{\psi_{ \pm}^{E_{1}}(x)} \psi_{ \pm}^{E_{2}}(x) d x=\delta\left(E_{1}-E_{2}\right)
$$

and completeness:

$$
\int \overline{\psi_{ \pm}^{E}(x)} \psi_{ \pm}^{E}\left(x^{\prime}\right) d E=\delta\left(x-x^{\prime}\right)
$$

Therefore, due to the Gelfand-Maurin spectral theorem one has

$$
\phi(x)=\sum_{ \pm} \int d E \psi_{ \pm}^{E}(x)\left\langle\psi_{ \pm}^{E} \mid \phi\right\rangle
$$


for any $\phi \in \mathcal{S}$, and the corresponding spectral resolution of the Hamiltonian has the following form:

$$
\widehat{H}=\sum_{ \pm} \int d E E\left|\psi_{ \pm}^{E}\right\rangle\left\langle\psi_{ \pm}^{E}\right|
$$

There is another family of energy eigenvectors directly related to $\psi_{ \pm}^{E}$. Due to (4.19) one has:

$$
\widehat{H} F\left[\psi_{ \pm}^{-E}\right]=E F\left[\psi_{ \pm}^{-E}\right]
$$

The Fourier transform of $\psi_{ \pm}^{E}$ is given by (cf. [13] and the Appendix):

$$
F\left[\psi_{ \pm}^{-E}\right](k)= \pm \frac{i}{2 \pi \sqrt{\gamma}} \exp \left[ \pm \frac{i \pi}{2}\left(i \frac{E}{\gamma}-\frac{1}{2}\right)\right] \Gamma\left(i \frac{E}{\gamma}+\frac{1}{2}\right)(k \pm i 0)^{-(i E / \gamma+1 / 2)} .
$$

One shows $[13]$ that $F\left[\psi_{ \pm}^{E}\right]$ are well defined tempered distributions for any $E \in \mathbb{R}$. Moreover,

$$
\int \overline{F\left[\psi_{ \pm}^{E_{1}}\right]}(x) F\left[\psi_{ \pm}^{E_{2}}\right](x) d x=\delta\left(E_{1}-E_{2}\right)
$$

and

$$
\int \overline{F\left[\psi_{ \pm}^{E}\right]}(x) F\left[\psi_{ \pm}^{E}\right]\left(x^{\prime}\right) d E=\delta\left(x-x^{\prime}\right)
$$

Hence, following the Gelfand-Maurin theorem, we have further spectral decompositions: for any $\psi \in \mathcal{S}$

$$
\psi(x)=\sum_{ \pm} \int d E F\left[\psi_{ \pm}^{-E}\right](x)\left\langle F\left[\psi_{ \pm}^{-E}\right] \mid \psi\right\rangle
$$

and for the Hamiltonian itself:

$$
\widehat{H}=\sum_{ \pm} \int d E E\left|F\left[\psi_{ \pm}^{-E}\right]\right\rangle\left\langle F\left[\psi_{ \pm}^{-E}\right]\right|
$$

\section{$7 \quad$ Analyticity of energy eigenvectors}

Let us continue the energy eigenvectors $\psi_{ \pm}^{E}$ and $F\left[\psi_{ \pm}^{-E}\right]$ into the energy complex plane $E \in \mathbb{C}$. It turns out [13] (see also the Appendix) that $\psi_{ \pm}^{E}$ has simple poles at $E=-E_{n}$, whereas $F\left[\psi_{ \pm}^{-E}\right]$ has simple poles at $E=+E_{n}$, with $E_{n}$ defined in (5.7). Therefore, the poles of energy eigenvectors considered as functions of the complex energy correspond exactly to the complex eigenvalues of $\widehat{H}$ which we found in Section 5. One easily computes the corresponding residues:

$$
\operatorname{Res}\left(\psi_{ \pm}^{E}(x) ;-E_{n}\right)=i(\mp 1)^{n} \sqrt{\frac{\gamma}{2 \pi}} \frac{\delta^{(n)}(x)}{n !}
$$

and

$$
\operatorname{Res}\left(F\left[\psi_{ \pm}^{-E}(x)\right] ;+E_{n}\right)= \pm \frac{\sqrt{\gamma}}{2 \pi}(\mp i)^{n+1} \frac{(-1)^{n}}{n !} x^{n}
$$


Hence, residues of $\psi_{ \pm}^{E}$ and $F\left[\psi_{ \pm}^{-E}\right]$ correspond, up to numerical factors, to the eigenvectors $f_{n}^{ \pm}(5.5)$ :

$$
\operatorname{Res}\left(\psi_{ \pm}^{E}(x) ;-E_{n}\right) \sim f_{n}^{-}
$$

and

$$
\operatorname{Res}\left(F\left[\psi_{ \pm}^{-E}(x)\right] ;+E_{n}\right) \sim f_{n}^{+} .
$$

Any function $\phi \in \mathcal{S} \subset L^{2}(\mathbb{R})$ gives rise to the following functions of energy:

$$
\mathbb{R} \ni E \longrightarrow\left\langle\psi_{ \pm}^{E} \mid \phi\right\rangle \in \mathbb{C},
$$

and

$$
\mathbb{R} \ni E \longrightarrow\left\langle F\left[\psi_{ \pm}^{-E}\right] \mid \phi\right\rangle \in \mathbb{C} .
$$

Let us introduce two important classes of functions [26]: a smooth function $f=f(E)$ is in the Hardy class from above $\mathcal{H}_{+}^{2}$ (from below $\mathcal{H}_{-}^{2}$ ) if $f(E)$ is a boundary value of an analytic function in the upper, i.e. $\operatorname{Im} E \geq 0$ (lower, i.e. $\operatorname{Im} E \leq 0$ ) half complex $E$-plane vanishing faster than any power of $E$ at the upper (lower) semi-circle $|E| \rightarrow \infty$. Now, define

$$
\Phi_{-}:=\left\{\phi \in \mathcal{S} \mid\left\langle\psi_{ \pm}^{E} \mid \phi\right\rangle \in \mathcal{H}_{-}^{2}\right\}
$$

and

$$
\Phi_{+}:=\left\{\phi \in \mathcal{S} \mid\left\langle F\left[\psi_{ \pm}^{-E}\right] \mid \phi\right\rangle \in \mathcal{H}_{+}^{2}\right\}
$$

Proposition $4 \Phi_{+} \cap \Phi_{-}=\{\emptyset\}$.

Proof. Clearly, if $\phi \in \Phi_{-}$, then $\left\langle\psi_{ \pm}^{E} \mid \phi\right\rangle$ is a smooth function of $E \in \mathbb{R}$. Suppose, that $\phi \in \Phi_{+}$, that is

$$
\left\langle F\left[\psi_{ \pm}^{-E}\right] \mid \phi\right\rangle=\left\langle\psi_{ \pm}^{-E} \mid F[\phi]\right\rangle \in \mathcal{H}_{+}^{2} .
$$

Now, due to the Paley-Wiener theorem [12] the inverse Fourier transform of $F[\phi]$

$$
F^{-1}[F[\phi]](E)=\frac{1}{\sqrt{2 \pi}} \int_{-\infty}^{\infty} F[\phi](t) e^{-i t E} d t,
$$

vanishes for $E>0$. Therefore, $\phi(E)=0$ for $E>0$, and hence $f(E)$ cannot be a smooth function of $E$.

Our main result consists in the following

Theorem 2 For any $\phi^{ \pm} \in \Phi_{ \pm}$one has

$$
\phi^{-}(x)=\sum_{n} f_{n}^{-}(x)\left\langle f_{n}^{+} \mid \phi^{-}\right\rangle
$$

and

$$
\phi^{+}(x)=\sum_{n} f_{n}^{+}(x)\left\langle f_{n}^{-} \mid \phi^{+}\right\rangle .
$$


Proof. Due to the spectral formula (6.11) one has, for $\phi^{-} \in \Phi_{-} \subset \mathcal{S}$ :

$$
\phi^{-}(x)=\sum_{ \pm} \int_{-\infty}^{\infty} d E \psi_{ \pm}^{E}(x)\left\langle\psi_{ \pm}^{E} \mid \phi^{-}\right\rangle .
$$

Now, since $\left\langle\psi_{ \pm}^{E} \mid \phi^{-}\right\rangle \in \mathcal{H}_{-}^{2}$, we may close the integration contour along the lower semi-circle $|E| \rightarrow \infty$. Hence, due to the residue theorem one obtains

$$
\phi^{-}(x)=-\left.2 \pi i \sum_{ \pm} \sum_{n} \operatorname{Res}\left(\psi_{ \pm}^{E}(x) ;-E_{n}\right)\left\langle\psi_{ \pm}^{E} \mid \phi^{-}\right\rangle\right|_{E=-E_{n}}
$$

Using the definition of $\psi_{ \pm}^{E}$

$$
\left\langle\psi_{ \pm}^{E} \mid \phi^{-}\right\rangle=\frac{1}{\sqrt{2 \pi \gamma}} \int \overline{x_{ \pm}^{-(i E / \gamma+1 / 2)}} \phi^{-}(x) d x=\frac{1}{\sqrt{2 \pi \gamma}} \int x_{ \pm}^{-(-i E / \gamma+1 / 2)} \phi^{-}(x) d x,
$$

one finds

$$
\left.\left\langle\psi_{ \pm}^{E} \mid \phi^{-}\right\rangle\right|_{E=-E_{n}}=\frac{1}{\sqrt{2 \pi \gamma}} \int x_{ \pm}^{n} \phi^{-}(x) d x
$$

Therefore, inserting into (7.12) the value of the residue given in (7.1) one gets finally

$$
\begin{aligned}
\phi^{-}(x) & =\sum_{n} \frac{\delta^{(n)}(x)}{n !} \int\left[(-1)^{n} x_{+}^{n}+x_{-}^{n}\right] \phi^{-}(x) d x=\sum_{n}(-1)^{n} \frac{\delta^{(n)}(x)}{n !} \int x^{n} \phi^{-}(x) d x \\
& =\sum_{n} f_{n}^{-}(x)\left\langle f_{n}^{+} \mid \phi^{-}\right\rangle .
\end{aligned}
$$

To prove (7.10) let us use another spectral formula (6.17): for any $\phi^{+} \in \Phi_{+} \subset \mathcal{S}$

$$
\phi^{+}(x)=\sum_{ \pm} \int_{-\infty}^{\infty} d E F\left[\psi_{ \pm}^{-E}\right](x)\left\langle F\left[\psi_{ \pm}^{-E}\right] \mid \phi^{-}\right\rangle .
$$

Now, since $\left\langle F\left[\psi_{ \pm}^{-E}\right] \mid \phi^{-}\right\rangle \in \mathcal{H}_{+}^{2}$, we may close the integration contour along the upper semicircle $|E| \rightarrow \infty$. Hence the residue theorem implies

$$
\phi^{+}(x)=+\left.2 \pi i \sum_{ \pm} \sum_{n} \operatorname{Res}\left(F\left[\psi_{ \pm}^{-E}(x)\right] ;+E_{n}\right)\left\langle F\left[\psi_{ \pm}^{-E}\right] \mid \phi^{+}\right\rangle\right|_{E=+E_{n}}
$$

Now, using once more the formula for $\psi_{ \pm}^{E}$ one finds

$$
\left.\left\langle F\left[\psi_{ \pm}^{-E}\right] \mid \phi^{+}\right\rangle\right|_{E=+E_{n}}=\frac{1}{\sqrt{2 \pi \gamma}}\left\langle F\left[x_{ \pm}^{n}\right] \mid \phi^{+}\right\rangle
$$


Hence, inserting the values of residues (7.2) into (7.17) and using the formula for $F\left[x_{ \pm}^{n}\right]$ (see (A.9)) one has

$$
\begin{aligned}
\phi^{+}(x) & =\frac{i}{\sqrt{2 \pi}} \sum_{n}(-1)^{n} \frac{x^{n}}{n !}\left[(-i)^{n+1}\left\langle F\left[x_{+}^{n}\right] \mid \phi^{+}\right\rangle-i^{n+1}\left\langle F\left[x_{-}^{n}\right] \mid \phi^{+}\right\rangle\right] \\
& =\frac{i}{\sqrt{2 \pi}} \sum_{n}(-1)^{n} \frac{x^{n}}{n !} \int\left[(-i)^{n+1} \overline{F\left[x_{+}^{n}\right](k)}-i^{n+1} \overline{F\left[x_{-}^{n}\right](k)}\right] \phi^{+}(k) d k \\
& =\frac{i}{2} \sum_{n}(-1)^{n} \frac{x^{n}}{n !}\left[(-i)^{n+1} i^{n}-i^{n+1}(-i)^{n}\right] \int \delta^{(n)}(k) \phi^{+}(k) d k \\
& =\sum_{n} f_{n}^{+}(x)\left\langle f_{n}^{-} \mid \phi^{+}\right\rangle,
\end{aligned}
$$

which ends the proof.

This way we have recovered (5.16) and (5.17). It is not surprising, due to the following

Proposition $5 \quad \Phi_{-}=\mathcal{Z}$ and $\Phi_{+}=\mathcal{D}$.

Corollary 1 We have two spectral decomposition of $\widehat{H}$ :

$$
\widehat{H}=\sum_{n} \bar{E}_{n}\left|f_{n}^{-}\right\rangle\left\langle f_{n}^{+}\right| \quad \text { on } \quad \Phi_{-},
$$

and

$$
\widehat{H}=\sum_{n} E_{n}\left|f_{n}^{+}\right\rangle\left\langle f_{n}^{-}\right| \quad \text { on } \quad \Phi_{+}
$$

\section{Resonances and the quantum damping}

Finally, let us turn to the evolution generated by the Hamiltonian (3.10). Obviously, it generates a 1-parameter unitary group

$$
U(t)=e^{-i \widehat{H} t},
$$

on $L^{2}(\mathbb{R})$. It follows from $(4.3)$ that

$$
\psi_{t}(x)=U(t) \psi(x)=e^{\gamma t / 2} \psi\left(e^{\gamma t} x\right) .
$$

The above formula is well defined for any $t \in \mathbb{R}$ and clearly, as we already showed, the theory is time-reversal invariant: if $\psi(t)$ solves the Schrödinger equation so does $\mathbf{T} \psi(t):=\psi(-t)$. Therefore, working in $L^{2}(\mathbb{R})$ we do not see any damping at all. Now, let us construct two natural Gelfand triplets:

$$
\Phi_{ \pm} \subset L^{2}(\mathbb{R}) \subset \Phi_{ \pm}^{\prime}
$$

If $\phi^{-} \in \Phi_{-}$, then

$$
\left\langle\psi_{ \pm}^{E} \mid U(t) \phi^{-}\right\rangle=\left\langle U^{*}(t) \psi_{ \pm}^{E} \mid \phi^{-}\right\rangle=e^{-i E t}\left\langle\psi_{ \pm}^{E} \mid \phi^{-}\right\rangle
$$


Hence $\phi^{-}(t) \in \Phi_{-}$only for $t \geq 0$. Similarly, if $\phi^{+} \in \Phi_{+}$, then $\phi^{+}(t) \in \Phi_{+}$only for $t \leq 0$. Therefore, the restriction of the unitary group $U(t)$ on $L^{2}(\mathbb{R})$ to $\Phi_{ \pm}$no longer defines a group. It gives rise to two semigroups:

$$
U_{-}(t): \Phi_{-} \longrightarrow \Phi_{-}, \quad \text { for } t \geq 0
$$

and

$$
U_{+}(t): \Phi_{+} \longrightarrow \Phi_{+}, \quad \text { for } t \leq 0 .
$$

Due to (7.20) and (7.21) one has:

$$
\phi^{-}(t)=U(t) \phi^{-}=\sum_{n} e^{-\gamma(n+1 / 2) t}\left|f_{n}^{-}\right\rangle\left\langle f_{n}^{+} \mid \phi^{-}\right\rangle,
$$

for $t \geq 0$, and

$$
\phi^{+}(t)=U(t) \phi^{+}=\sum_{n} e^{\gamma(n+1 / 2) t}\left|f_{n}^{+}\right\rangle\left\langle f_{n}^{-} \mid \phi^{+}\right\rangle
$$

for $t \leq 0$. We stress that $\phi_{t}^{-}\left(\phi_{t}^{+}\right)$does belong to $L^{2}(\mathbb{R})$ also for $t<0(t>0)$. However, $\phi_{t}^{-} \in \Phi_{-}\left(\phi_{t}^{+} \in \Phi_{+}\right)$only for $t \geq 0(t \leq 0)$. This way the irreversibility enters on a purely Hamiltonian level by restricting dynamics to the dense subspace $\Phi_{ \pm}$of $L^{2}(\mathbb{R})$.

Clearly, formulae (8.7) and (8.8) are quantum analogues of the classical damping laws:

$$
x(t)=e^{-\gamma t} x, \quad t \geq 0,
$$

and

$$
p(t)=e^{+\gamma t} p, \quad t \leq 0
$$

Finally, let us recall that the time reversal operator $\mathbf{T}$ establishes an isomorphism between $\Phi_{-}$and $\Phi_{+}$. Therefore, each solution

$$
\phi_{t}^{-}=U_{-}(t) \phi^{-}
$$

with $\phi^{-} \in \Phi_{-}$is mapped into

$$
\mathbf{T}\left(\phi_{t}^{-}\right)=U_{-}(-t) \mathbf{T}\left(\phi^{-}\right)=U_{+}(t) \mathbf{T}\left(\phi^{-}\right), \quad t \leq 0 .
$$

Conversely, any solution

$$
\phi_{t}^{+}=U_{+}(t) \phi^{+}
$$

with $\phi^{+} \in \Phi_{+}$is mapped into

$$
\mathbf{T}\left(\phi_{t}^{+}\right)=U_{+}(-t) \mathbf{T}\left(\phi^{+}\right)=U_{-}(t) \mathbf{T}\left(\phi^{+}\right), \quad t \geq 0 .
$$

Summarizing, quantum dynamics is irreversible on $\Phi_{-}$and $\Phi_{+}$. This irreversibility is caused by quantum damping, or, equivalently, by the presence of resonant states $f_{n}^{ \pm}(5.5)$. It should be stressed that it is not an energy that is dissipated. Clearly, the Hamiltonian (3.10) can 
not be interpreted as a system energy - it was used to define a Hamiltonian dynamics of the enlarged system on $L^{2}(\mathbb{R})$. The quantum damped system is not defined on the entire $L^{2}(\mathbb{R})$ but rather on a dense subset $\Phi_{-} \subset L^{2}(\mathbb{R})$. As we saw it imposes the restriction upon the time evolution such that it is defined only for positive $t$. A quantum damping may be seen as follows: let $\phi_{0}^{-} \in \Phi_{-}$be an initial state then the probability density for a particle position evolves in time as follows:

$$
p_{t}(x)=\left|\phi_{t}^{-}(x)\right|^{2}=e^{\gamma t}\left|\phi_{0}^{-}\left(e^{\gamma t} x\right)\right|^{2}=e^{\gamma t} p_{0}\left(e^{\gamma t} x\right),
$$

and hence in the limit $t \longrightarrow+\infty$, one finds $p_{t}(x) \longrightarrow \delta(x)$. Indeed, for any $\epsilon>0$

$$
\int_{-\epsilon}^{\epsilon} p_{t}(x) d x=\int_{-\epsilon e^{\gamma t}}^{\epsilon e^{\gamma t}} p_{0}(x) d x \longrightarrow 1
$$

for $t \longrightarrow+\infty$. Clearly, it corresponds to the classical behavior $x(t)=e^{-\gamma t} x_{0} \longrightarrow 0$.

In a forthcoming paper we are going to show that also more complicated damped systems, e.g. the damped harmonic oscillator, give rise to irreversible dynamics.

\section{A Appendix}

The regular tempered distribution $x_{+}^{\lambda}($ with $\lambda \in \mathbb{C}$ ) given by

$$
\left\langle\phi \mid x_{+}^{\lambda}\right\rangle:=\int_{0}^{\infty} x^{\lambda} \phi(x) d x
$$

for any $\phi \in \mathcal{S}$, is well defined for $\operatorname{Re} \lambda>-1$. However, it may be easily extended to the region $\operatorname{Re} \lambda>-2$ due to the following regularization formula:

$$
\int_{0}^{\infty} x^{\lambda} \phi(x) d x=\int_{0}^{1} x^{\lambda}[\phi(x)-\phi(0)] d x+\int_{1}^{\infty} x^{\lambda} \phi(x) d x+\frac{\phi(0)}{\lambda+1},
$$

which holds for $\lambda \neq-1$. In the same way one may extend the distribution $x_{+}^{\lambda}$ to the region $\operatorname{Re} \lambda>-n-1$ using the formula

$$
\begin{aligned}
\int_{0}^{\infty} x^{\lambda} \phi(x) d x & =\int_{0}^{1} x^{\lambda}\left[\phi(x)-\phi(0)-x \phi^{\prime}(0)-\ldots-\frac{x^{n-1}}{(n-1) !} \phi^{(n-1)}(0)\right] d x \\
& +\int_{1}^{\infty} x^{\lambda} \phi(x) d x+\sum_{k=1}^{n} \frac{\phi^{(k-1)}(0)}{(k-1) !(\lambda+k)}
\end{aligned}
$$

which holds for $\lambda \neq-1,-2, \ldots,-n$. The above formula shows that $\left\langle\phi \mid x_{+}^{\lambda}\right\rangle$ as a function of $\lambda \in \mathbb{C}$ has simple poles at $\lambda=-1,-2, \ldots$, and the corresponding residue at $\lambda=-k$ equals $\phi^{(k-1)}(0) /(k-1)$ !. 
Using the same arguments one shows that the distribution $x_{-}^{\lambda}$ may be extended to the region $\operatorname{Re} \lambda>-n-1$ via:

$$
\begin{aligned}
\int_{-\infty}^{0} x^{\lambda} \phi(x) d x & =\int_{0}^{\infty} x^{\lambda} \phi(-x) d x \\
& =\int_{1}^{\infty} x^{\lambda}\left[\phi(-x)-\phi(0)+x \phi^{\prime}(0)-\ldots-\frac{(-1)^{n-1} x^{n-1}}{(n-1) !} \phi^{(n-1)}(0)\right] d x \\
& +\int_{1}^{\infty} x^{\lambda} \phi(x) d x+\sum_{k=1}^{n} \frac{(-1)^{k-1} \phi^{(k-1)}(0)}{(k-1) !(\lambda+k)}
\end{aligned}
$$

which holds for $\lambda \neq-1,-2, \ldots,-n$. Hence, $\left\langle\phi \mid x_{-}^{\lambda}\right\rangle$ has simple poles at $\lambda=-1,-2, \ldots$, and the corresponding residue at $\lambda=-k$ equals $(-1)^{k-1} \phi^{(k-1)}(0) /(k-1)$ !.

The Fourier transforms of $x_{ \pm}^{\lambda}$

$$
F\left[x_{ \pm}^{\lambda}\right](k)=\frac{1}{\sqrt{2 \pi}} \int e^{i k x} x_{ \pm}^{\lambda} d x
$$

are given by the following formula [13]

$$
F\left[x_{ \pm}^{\lambda}\right](k)= \pm \frac{i}{\sqrt{2 \pi}} e^{ \pm i \lambda \pi / 2} \Gamma(\lambda+1)(k+i 0)^{-\lambda-1},
$$

where $(k \pm i 0)^{\alpha}$ is a distribution defined by:

$$
(k \pm i 0)^{\alpha}=k_{+}^{\alpha}+e^{ \pm i \alpha \pi} k_{-}^{\alpha} .
$$

Due to the Euler $\Gamma$-function the formula (A.6) has single poles at $\lambda=-1,-2, \ldots$ Note, that although both $k_{+}^{\alpha}$ and $k_{-}^{\alpha}$ have poles at $\alpha=-1,-2, \ldots$, the distribution $(k \pm i 0)^{\alpha}$ is well defined for all $\alpha \in \mathbb{C}$. Indeed

$$
\lim _{\alpha \rightarrow-n}(k \pm i 0)^{\alpha}=\lim _{\alpha \rightarrow-n}\left(k_{+}^{\alpha}+(-1)^{n} k_{-}^{\alpha}\right)
$$

and, due to (A.3) and (A.4), the singular parts of $k_{+}^{\alpha}$ and $k_{-}^{\alpha}$, at $\alpha=-n$, cancel out. In particular, for $\lambda=n \in \mathbb{N}$, one obtains (cf. [13])

$$
F\left[x_{ \pm}^{n}\right](k)=\frac{1}{\sqrt{2 \pi}}\left[( \pm i)^{n+1} n ! k^{-n-1}+(\mp i)^{n} \pi \delta^{(n)}(k)\right] .
$$

\section{Acknowledgments}

I would like to thank Jȩdrzej Śniatycki for very interesting discussions and his warm hospitality during my stay in Calgary and Andrzej Kossakowski for introducing this problem to me and for many interesting and stimulating discussions. This work was partially supported by the Polish State Committee for Scientific Research (KBN) Grant no 2P03B01619. 


\section{References}

[1] L.A. Khalfin, JETP Lett. 5 (1972) 388

[2] C.G Hegerfeldt, Phys. Rev. Lett. 72 (1994) 596

[3] A. Bohm, H.-D. Doebner, P. Kielanowski, Irreversibility and Causality, Semigroups and Rigged Hilbert Spaces, Lecture Notes in Physics 504, Springer, Berlin, 1998.

[4] A. Bohm and M. Gadella, Dirac Kets, Gamov Vectors and Gelfand Triplets, Lecture Notes in Physics 348, Springer, Berlin, 1989

[5] I. Antoniou and I. Prigogine, Physica A 192 (1993) 443

[6] I.M. Gelfand and N.Y. Vilenkin, Generalized Functions, Vol. IV, Academic Press, New York, 1964.

[7] K. Maurin, General Eigenfunction Expansion and Unitary Representations of Topological Groups, PWN, Warszawa, 1968.

[8] G. Parravicini, V. Gorini and E.C.G. Sudarshan, J. Math. Phys. 21 (1980) 2208

[9] S. Albeverio, L.S. Ferreira and L. Streit, eds. Resonances - Models and Phenomena, Lecture Notes in Physics 211, Springer, Berlin, 1984

[10] E. Brandas and N. Elander, eds. Resonances, Lecture Notes in Physics 325, Springer, Berlin, 1989

[11] M. Reed and B. Simon, Methods of Modern Mathematical Physics. Vol. III. Scattering Theory, Academic, New York, 1979

[12] K. Yosida, Functional Analysis, Springer, Berlin, 1978

[13] I.M. Gelfand and G.E. Shilov, Generalized functions, Vol. I, Academic Press, New York, 1966

[14] L.S. Pontriagin, V.G. Boltańskij, R.V. Gamkrelidze, E.F. Miscenko, The Mathematical Theory of Optimal Processes, Wiley, New York, 1962

[15] D.F. Walls, Nature, 306 (1983) 141

[16] L.D. Landau and E.M. Lifshitz, Quantum Mechanics, Pergamon, London, 1958

[17] G. Barton, Ann. Phys. 166 (1986) 322

[18] N.L. Balazs and A. Voros, Ann. Phys. 199 (1990) 123

[19] M. Castagnino, R. Diener, L. Lara and G. Puccini, Int. Jour. Theor. Phys. 36 (1997) 2349

[20] D. Chruściński, Open Sys. Information Dyn. 9 (2002) 207 (available as LANL e-print math-ph/0206009) 
[21] D. Chruściński, Wigner function for damped systems, LANL e-print math-ph/0209008

[22] E. P. Wigner, Unitary representation of the Inhomogeneous Lorentz Group Including Reflections, in Group Theoretical Methods in Elementary Particle Physics, F. Gúrsay (ed.), Gordon and Breach, Science Publisher, New York, 1967

[23] A. Kossakowski, private communication

[24] R.P. Kanwal, Generalized Functions: Theory and Techniques, Mathematics in Science and Engineering 177, Academic Press, New York, 1983

[25] C.G. Bollini and L.E. Oxman, Phys. Rev. A 47 (1993) 2339

[26] P.L. Duren, Theory of $\mathcal{H}^{p}$ Spaces, Academic Press, New York, 1970 